\title{
Determination of bulk domain structure and magnetization processes in bcc ferromagnetic alloys: Analysis of magnetostriction in $\mathrm{Fe}_{83} \mathrm{Ga}_{17}$
}

\author{
Yangkun He, ${ }^{1}$ J. M. D. Coey, ${ }^{1,2}$ Rudolf Schaefer, ${ }^{3}$ and Chengbao Jiang ${ }^{1, *}$ \\ ${ }^{1}$ School of Materials Science and Engineering, Beihang University, Beijing 100191, People's Republic of China \\ ${ }^{2}$ School of Physics, Trinity College, Dublin 2, Ireland \\ ${ }^{3}$ Leibniz Institute for Solid State and Materials Research (IFW), Dresden, Germany
}

(Received 1 August 2017; published 23 January 2018)

\begin{abstract}
The ground state of macroscopic samples of magnetically ordered materials is a domain state because of magnetostatic energy or entropy, yet we have limited experimental means for imaging the bulk domain structure and the magnetization process directly. The common methods available reveal the domains at the surface or in electron- or X-ray transparent lamellae, not those in the bulk. The magnetization curve just reflects the vector sum of the moments of all the domains in the sample, but magnetostriction curves are more informative. They are strongly influenced by the domain structure in the unmagnetized state and its evolution during the magnetization process in an applied field. Here we report a method of determining the bulk domain structure in a cubic magnetostrictive material by combining magneto-optic Kerr microscopy with magnetostriction and magnetization measurements on single crystals as a function of applied field. We analyze the magnetostriction of $\mathrm{Fe}_{83} \mathrm{Ga}_{17}$ crystals in terms of a domain structure that is greatly influenced by sample shape and heat treatment. Saturation magnetostriction measurements are used to determine the fraction of domains orientated along the three $\langle 100\rangle$ axes in the initial state. Domain wall motion and rotation process have characteristic signatures in the magnetostriction curves, including those associated with the $\Delta E$ effect and domain rotation through a $\langle 110\rangle$ auxetic direction.
\end{abstract}

DOI: 10.1103/PhysRevMaterials.2.014412

\section{INTRODUCTION}

Magnetically ordered materials with a net spontaneous magnetization generally adopt a multidomain structure in their ground state in order to minimize the magnetostatic self-energy associated with the $H$ field they produce [1,2]. Alternatively, the self-energy can be associated with the magnetization of the material in its own demagnetizing field - the two formulations are equivalent [3]. Domains are the bridge between the microstructure of a specimen of a given shape and its macroscopic magnetic properties such as anisotropy, saturation field, and magnetostriction. Domain structure is the key to understanding the macroscopic magnetic properties in an unsaturated state; as soon as a ferromagnet is magnetically saturated, it becomes a monodomain.

There are several well-established methods for observing domains. First came the Bitter method [1], based on the patterning of a drop of magnetic colloid by the stray field at a polished specimen surface. Magnetic force microscopy (MFM) is an alternative way to image the stray field or the magnetic "charge" distribution at the surface [4]. However, the most commonly used method is magneto-optic Kerr effect microscopy, using polarized light in reflection in a metallographic microscope [2]. Scanning electron microscopy with polarization analysis (SEMPA) [5] similarly images the domain configuration at the surface, but with higher resolution. Domains in thin, electrontransparent lamellae can be imaged by Lorentz microscopy in a transmission electron microscope (TEM) or by electron holography. Domains in x-ray transparent specimens can be

*jiangcb@buaa.edu.cn imaged by $\mathrm{x}$-ray topography, which depends on magnetostrictive distortion [6], or anomalous magnetic x-ray scattering [7]. X-ray scattering lengths for $3 d$ metals are of order $10 \mu \mathrm{m}$. The only method that is available for imaging domains in the bulk is neutron topography [8], especially using neutron darkfield microscopy $[9,10]$, which depends on magnetic neutron scattering - a weak interaction leading to neutron scattering and absorption lengths in solids or order $0.1 \mathrm{~m}$. Hence the neutrons can probe bulk samples. However, these methods are not in widespread use because they depend on intense neutron or synchrotron x-ray beams at large-scale facilities.

Magnetostriction is the basis of important applications in precision machinery, microactuators, sonar transducers, stress sensors, and smart components. It is the result of domain reorientation in an applied field, and its magnitude usually depends on the difference between the microscale domain structure in the unmagnetized and saturated states [11]. Here we are interested in Galfenol, a cubic Fe-Ga alloy with high magnetostriction and favorable mechanical properties that is important for many applications. Domains in this material have been studied by the Bitter method [12], Kerr microscopy [13-15], MFM [13,16-20], and Lorentz microscopy [21], all of which image domains at the surface, or in thin lamellae; there is also a neutron scattering study of magnetic heterogeneities [22]. For cubic materials such as Fe-Ga with $\langle 100\rangle$ easy axes, $90^{\circ}$ wall motion and domain reorientation processes contribute significantly to magnetostriction, while magnetization processes that involve the motion of $180^{\circ}$ domain walls have no effect on sample dimensions $[23,24]$ because the strain does not depend on the sign of the magnetization. Likewise, domains that remain magnetized in a plane normal to 
the magnetostriction measurement direction have practically no effect. Whenever the sample is pressed [25], prestressed [26-29], or annealed in a magnetic field [30], the initial domain structure adapts to minimize the magnetoelastic energy, and therefore the change in magnetostriction at saturation can vary greatly according to the initial state. The recently reported "non-Joulian" magnetostriction [12,31] appears to be related to a special domain structure found at the surface of certain thin disk-shaped single crystals [32]. It is therefore desirable to have an overview of the bulk domain structure in Fe-Ga and its dependence on crystal shape.

In this paper we use Kerr microscopy in combination with magnetostriction measurements to investigate the domains in $\mathrm{Fe}_{83} \mathrm{Ga}_{17}$, both in the unmagnetized state where they are greatly influenced by sample shape, and during the approach to saturation. Isotropically distributed domains are found in equiaxed bulk samples, but the anisotropic domain structures that appear in thin samples can be quantified by measuring the magnetostriction in different directions and the domain structure is explicitly taken into account when calculating the cubic magnetostriction constants $\lambda_{100}$ and $\lambda_{111}$. As a check, these intrinsic magnetostriction constants are deduced independently from the angular dependence of the strain observed at saturation, which does not require any knowledge of the initial domain structure. We find that the magnetostriction in $\mathrm{Fe}_{83} \mathrm{Ga}_{17}$ is generally volume conserving.

TABLE I. Summary of saturation magnetostriction data on $\mathrm{Fe}_{83} \mathrm{Ga}_{17}$ crystals. The error on the mean of $\Sigma \lambda_{i}$, the sum of the magnetostriction in the three principal directions, is the variance of the experimental data. Except in the last case, the (001) plane data in the table were measured in the middle parts of the crystals. "Quenched" and "slowly cooled" refer to the heat treatment specified in Sec. II. Other samples were furnace-cooled after crystal growth at an intermediate cooling rate.

\begin{tabular}{|c|c|c|c|c|c|c|c|c|c|c|c|}
\hline \multirow[b]{2}{*}{ Sample } & \multirow[b]{2}{*}{$H$ direction } & \multicolumn{6}{|c|}{ Magnetostriction (ppm) } & \multirow[b]{2}{*}{$n_{x}$} & \multirow[b]{2}{*}{$n_{y}$} & \multirow[b]{2}{*}{$n_{z}$} & \multirow[b]{2}{*}{$\mathcal{N}$} \\
\hline & & $\lambda_{[100]}$ & $\lambda_{[110]}$ & $\lambda_{[010]}$ & $\lambda_{[1-10]}$ & $\lambda_{[001]}$ & $\Sigma \lambda_{i}$ & & & & \\
\hline$\phi 5 \times 5 \mathrm{~mm}$ & $\begin{array}{l}{[100]} \\
{[110]}\end{array}$ & $\begin{array}{r}198 \\
58\end{array}$ & $\begin{array}{l}52 \\
54\end{array}$ & $\begin{array}{c}-92 \\
-\end{array}$ & - & $\begin{array}{l}-92 \\
-99\end{array}$ & $\begin{array}{r}14 \\
-1\end{array}$ & $33 \%$ & $33 \%$ & $33 \%$ & 0.308 \\
\hline cubic $5 \times 5 \times 5 \mathrm{~mm}$ & $\begin{array}{l}{[100]} \\
{[110]}\end{array}$ & $\begin{array}{r}207 \\
52\end{array}$ & $\begin{array}{l}58 \\
53\end{array}$ & $\begin{array}{c}-100 \\
-\end{array}$ & - & $\begin{array}{r}-100 \\
-99\end{array}$ & $\begin{array}{r}7 \\
-1\end{array}$ & $33 \%$ & $33 \%$ & $33 \%$ & 0.333 \\
\hline$\phi 5 \times 1.5 \mathrm{~mm}$ & $\begin{array}{l}{[100]} \\
{[110]}\end{array}$ & $\begin{array}{r}215 \\
62\end{array}$ & $\begin{array}{l}65 \\
72\end{array}$ & $\begin{array}{c}-82 \\
-\end{array}$ & - & $\begin{array}{l}-120 \\
-114\end{array}$ & $\begin{array}{l}13 \\
21\end{array}$ & $29 \%$ & $29 \%$ & $42 \%$ & 0.596 \\
\hline $10.6 \times 10.6 \times 2.4 \mathrm{~mm}$ & $\begin{array}{l}{[100]} \\
{[110]} \\
{[001]}\end{array}$ & $\begin{array}{r}200 \\
80 \\
-40\end{array}$ & $\begin{array}{r}75 \\
85 \\
-\end{array}$ & $\begin{array}{l}-50 \\
- \\
-40\end{array}$ & $\begin{array}{l}- \\
73 \\
-\end{array}$ & $\begin{array}{r}-150 \\
-150 \\
90\end{array}$ & $\begin{array}{r}0 \\
8 \\
10\end{array}$ & $20 \%$ & $20 \%$ & $60 \%$ & 0.688 \\
\hline $10.6 \times 10.6 \times 2.4 \mathrm{~mm}$ quenched & $\begin{array}{l}{[100]} \\
{[010]} \\
{[001]} \\
{[110]} \\
{[1-10]}\end{array}$ & $\begin{array}{l}161 \\
-85 \\
-80 \\
- \\
-\end{array}$ & $\begin{array}{r}- \\
- \\
-78 \\
44 \\
35\end{array}$ & $\begin{array}{l}-80 \\
160 \\
-80 \\
- \\
-\end{array}$ & $\begin{array}{c}- \\
- \\
-80 \\
35 \\
43\end{array}$ & $\begin{array}{l}-75 \\
-75 \\
155 \\
-75 \\
-76\end{array}$ & $\begin{array}{c}6 \\
0 \\
-5 /-3 \\
4 \\
2\end{array}$ & $35 \%$ & $34 \%$ & $31 \%$ & 0.688 \\
\hline & $\begin{array}{l}{[100]} \\
{[010]}\end{array}$ & $\begin{array}{r}110 \\
-110\end{array}$ & $\begin{array}{l}- \\
-\end{array}$ & $\begin{array}{r}-111 \\
113\end{array}$ & $\begin{array}{l}- \\
-\end{array}$ & $\begin{array}{l}4 \\
2\end{array}$ & $\begin{array}{l}3 \\
5\end{array}$ & & & & \\
\hline $10.6 \times 10.6 \times 2.4 \mathrm{~mm}$ slowly cooled & $\begin{array}{r}{[001]} \\
{[110]} \\
{[1-10]}\end{array}$ & $\begin{array}{c}-113 \\
- \\
-\end{array}$ & $\begin{array}{r}-105 \\
12 \\
2\end{array}$ & $\begin{array}{c}-113 \\
- \\
-\end{array}$ & $\begin{array}{r}-108 \\
3 \\
16\end{array}$ & $\begin{array}{l}226 \\
-5 \\
-6\end{array}$ & $\begin{array}{r}0 / 13 \\
10 \\
12\end{array}$ & $49 \%$ & $49 \%$ & $2 \%$ & 0.688 \\
\hline$\phi 5 \times 0.88 \mathrm{~mm}$ & $\begin{array}{l}{[100]} \\
{[110]}\end{array}$ & $\begin{array}{r}250 \\
88\end{array}$ & $\begin{array}{l}78 \\
85\end{array}$ & $\begin{array}{c}-56 \\
-\end{array}$ & - & $\begin{array}{l}-176 \\
-175\end{array}$ & $\begin{array}{r}18 \\
-15\end{array}$ & $20 \%$ & $20 \%$ & $60 \%$ & 0.716 \\
\hline$\phi 5 \times 0.5 \mathrm{~mm}$ quenched & $\begin{array}{l}{[100]} \\
{[010]} \\
{[001]}\end{array}$ & $\begin{array}{r}35 \\
-230 \\
-230\end{array}$ & $\begin{array}{l}- \\
- \\
-\end{array}$ & $\begin{array}{r}-40 \\
220 \\
-40\end{array}$ & $\begin{array}{l}- \\
- \\
-\end{array}$ & $\begin{array}{r}0 \\
8 \\
-\quad\end{array}$ & $\begin{array}{l}-5 \\
-2 \\
-\end{array}$ & $85 \%$ & $15 \%$ & $0 \%$ & 0.816 \\
\hline$\phi 5 \times 0.5 \mathrm{~mm}$ slowly cooled & $\begin{array}{l}{[100]} \\
{[010]} \\
{[001]}\end{array}$ & $\begin{array}{r}155 \\
-100 \\
-100\end{array}$ & $\begin{array}{l}- \\
- \\
-\end{array}$ & $\begin{array}{r}-95 \\
155 \\
-100\end{array}$ & $\begin{array}{l}- \\
- \\
-\end{array}$ & $\begin{array}{l}-47 \\
-45 \\
-\end{array}$ & $\begin{array}{l}13 \\
10 \\
-\end{array}$ & $40 \%$ & $40 \%$ & $20 \%$ & 0.816 \\
\hline$\phi 7.26 \times 0.54 \mathrm{~mm}$ & $\begin{array}{l}{[100]} \\
{[010]}\end{array}$ & $\begin{array}{r}200 \\
-70\end{array}$ & - & $\begin{array}{r}-80 \\
190\end{array}$ & - & - & - & $26 \%$ & $30 \%$ & $44 \%$ & 0.856 \\
\hline$\phi 7 \times 0.1 \mathrm{~mm}$ (middle) & $\begin{array}{l}{[100]} \\
{[010]} \\
{[110]}\end{array}$ & $\begin{array}{r}210 \\
5 \\
50\end{array}$ & $\begin{array}{l}65 \\
28 \\
45\end{array}$ & $\begin{array}{r}-120 \\
80 \\
45\end{array}$ & $\begin{array}{l}- \\
- \\
40\end{array}$ & $\begin{array}{l}- \\
- \\
-\end{array}$ & $\begin{array}{l}- \\
- \\
-\end{array}$ & $0 \%$ & $57 \%$ & $43 \%$ & 0.968 \\
\hline$\phi 7 \times 0.1 \mathrm{~mm}$ (edge) & $\begin{array}{l}{[100]} \\
{[010]} \\
{[110]} \\
\quad \text { Mean }\end{array}$ & $\begin{array}{l}162 \\
-40 \\
-\end{array}$ & $\begin{array}{r}24 \\
-10 \\
12\end{array}$ & $\begin{array}{l}-40 \\
161 \\
-\end{array}$ & $\begin{array}{l}- \\
- \\
2\end{array}$ & $\begin{array}{l}- \\
- \\
-\end{array}$ & $\begin{array}{c}- \\
- \\
- \\
6 \pm 8\end{array}$ & $21 \%$ & $79 \%$ & $0 \%$ & 0.968 \\
\hline
\end{tabular}


Our work represents a fresh approach to determining the bulk domain structure and investigating the magnetization process for highly magnetostrictive cubic materials.

\section{EXPERIMENTAL METHODS}

Precursor ingots of $\mathrm{Fe}_{83} \mathrm{Ga}_{17}$ were prepared from $99.99 \%$ $\mathrm{Fe}$ and Ga by arc melting. All ingots were annealed at $1000^{\circ} \mathrm{C}$ for $10 \mathrm{~h}$ under argon, and single crystals were then grown from them in an optical image furnace using floating-zone recrystallization at a growth rate of $4 \mathrm{~mm} / \mathrm{h}$. Oriented crystals were cut and polished for magnetostriction measurements. Some crystals were in the as-grown, furnace-cooled condition. Others were subsequently heat treated in vacuum at $760{ }^{\circ} \mathrm{C}$ for $30 \mathrm{~min}$ and then quenched in water or slowly cooled at $10{ }^{\circ} \mathrm{C}$ per min to $200^{\circ} \mathrm{C}$.

In order to investigate shape effects, we prepared a series of disk- and plate-shaped $\mathrm{Fe}_{83} \mathrm{Ga}_{17}$ crystals with different demagnetizing factors $\mathcal{N}$. We cut four [001] single-crystal specimens $5 \mathrm{~mm}$ in diameter with thicknesses $0.5,0.88$, 1.5 , and $5.0 \mathrm{~mm}$, two [001] single-crystal specimens $7 \mathrm{~mm}$ in diameter with thicknesses 0.10 and $0.54 \mathrm{~mm}$, and a rectangular and a cube-shaped [001] single-crystal specimen $10.6 \times 10.6 \times 2.43 \mathrm{~mm}^{3}$ and $5 \times 5 \times 5 \mathrm{~mm}^{3}$. Altogether we have studied a dozen different samples, including different heat treatments for the rectangular and $\phi 5 \times 0.5$ crystals and different areas analyzed for the thin $\phi 7 \times 0.1$ disk. The samples are listed in Table I.

Magnetostriction in the (001) plane was measured using the standard strain gauge method, with dc excitation in a magnetic field produced by a $1.2 \mathrm{~T}$ electromagnet, or a $1.0 \mathrm{~T}$ Multimag permanent-magnet variable flux source, where the field can be rotated in the plane perpendicular to the axis of the magnet bore without moving the sample [33]. The meander of the strain gauge is $200 \mu \mathrm{m}$ wide. Magnetostriction perpendicular to the (001) plane for some of the thin samples was measured by capacitance dilatometry. Detailed experimental methods can be found in the Appendix.

The surface domain structures were observed by magnetooptic Kerr effect (MOKE) microscopy. To get an overview of the complete specimens, a low-resolution type microscope with separated illumination and observation light paths was used, while high-resolution domain observations were performed in a conventional wide-field Kerr microscope. Videos were recorded as the applied magnetic field was varied and images were captured in different critical magnetic fields. Magnetization curves were calculated from the Kerr images, or measured directly using a Physical Property Measurement System (PPMS).

Adequate polishing is essential to observe domains representative of the $\mathrm{Fe}-\mathrm{Ga}$ surface because they are sensitive to surface strain [13]. Therefore the crystal surfaces were polished with an additional step using colloidal amorphous silica after conventional polishing in order to achieve good images in the Kerr microscope.

\section{RESULTS}

First, in Fig. 1 the domain structure is studied on a $\phi$ $5 \times 5 \mathrm{~mm}$ cylinder. There are many $40-\mu \mathrm{m}$-wide zigzag shaped domains at the surface. The domains are in-plane twins in the
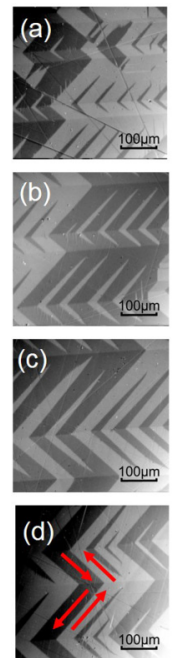

(k)
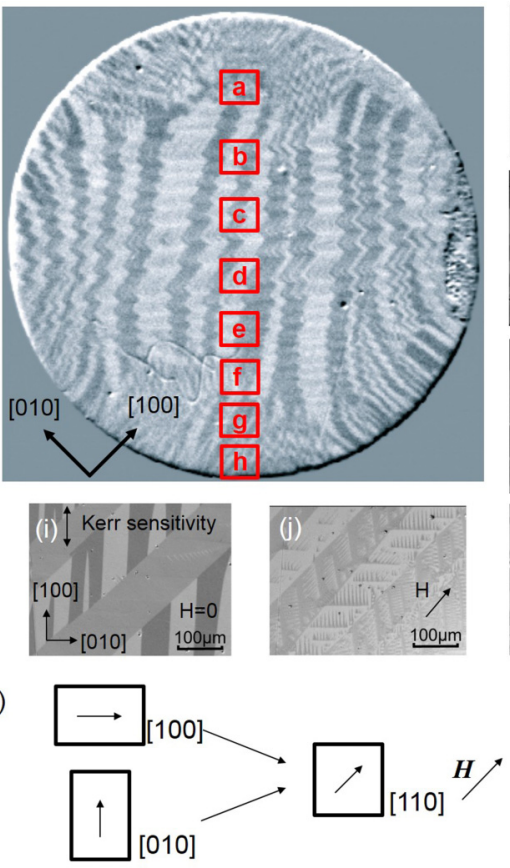

FIG. 1. Overview Kerr microscopy image of $\phi 5 \times 5 \mathrm{~mm}$ sample. (a) to (h) High-resolution images of spots a to h, respectively. The domains are in-plane twins magnetized in the [100] or [010] directions. In a field of $18 \mathrm{mT}$ applied along [110], the change of contrast in (i) and (j) shows that magnetization process involves domain rotation, as illustrated in (k). Note that for (i), (j), and (k), the sample is rotated by $45^{\circ}$ compared to all the other pictures.

[100] and [010] directions, which is a stress-free structure that releases the magnetostrictive strain [12]. All domains are parallel to the surface, and no perpendicular domains are observed. In the field of $18 \mathrm{mT}$ applied along [110], the change of contrast in Figs. 1(i) and 1(j) shows that the magnetization process involves domain rotation as illustrated in Fig. 1(k).

Magnetostriction measured with strain gauges along the three easy directions is shown in Fig. 2(a), when the field is applied along [100]. The figure shows that $\lambda_{[100]}=198 \mathrm{ppm}$ (parts per million), while $\lambda_{[010]}$ and $\lambda_{[001]}$ are both $-92 \mathrm{ppm}$. The data indicate that in a bulk isotropic single crystal, domains contribute uniformly in each easy direction, since $\lambda_{\|} \approx-2 \lambda_{\perp}$ with fractions $\left(n_{x}, n_{y}, n_{z}\right)=(1 / 3,1 / 3,1 / 3)$. The domains are equally likely to lie along any of the three easy axes in the crystal. Therefore, although we cannot directly observe the perpendicular domains on the surface in the Kerr microscope, we can infer that they are buried inside. A schematic of the twinlike domain structure of a cubic isotropic sample with $\mathcal{N} \approx 0.33$ is shown in Fig. 3 .

Figure 2(b) shows the magnetostriction measured in three orthogonal directions, [110], [1-10], and [001], when the field is applied along [110]. Here we find that the in-plane directions expand by 54 and $44 \mathrm{ppm}$ parallel and perpendicular to the applied magnetic field, while the [001] direction shrinks by $99 \mathrm{ppm}$. The intrinsic tetragonal and trigonal magnetostriction constants $(3 / 2) \lambda_{100}$ and $3 \lambda_{111}$ are best determined by rotation of the applied field in a (001) plane with strain gauges set in the [100] and [110] directions; this is done using the $1 \mathrm{~T}$ Multimag permanent magnet variable flux source [33], to 

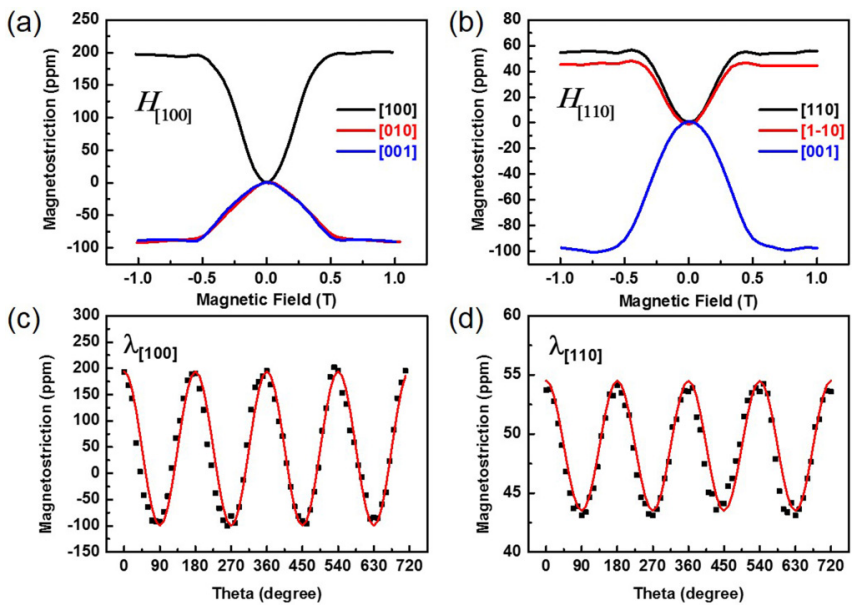

FIG. 2. Magnetostriction of a $\phi 5 \times 5 \mathrm{~mm} \mathrm{Fe}_{83} \mathrm{Ga}_{17}$ single crystal. (a) Magnetostriction in three orthogonal directions [100], [010], and [001] when the magnetic field $H$ is applied along [100]. (b) Magnetostriction in three orthogonal directions [110], [1-10], and [001] when the magnetic field $H$ is applied along [110]. In (c) and (d) a $1 \mathrm{~T}$ field is rotated in-plane while magnetostriction is measured along [100] and [110], respectively.

saturate the magnetization with results shown in Figs. 2(c) and $2(d)$. The fit to the peak to peak variation of the saturation magnetostriction [11] gives values of $(3 / 2) \lambda_{100}=280 \mathrm{ppm}$ and $3 \lambda_{111}=27 \mathrm{ppm}$, in agreement with previous reports for this composition [34]. Estimated error is $\pm 3 \%$. The advantage of measuring at saturation is that the magnetostriction constant can be obtained without knowing the initial domain state [11]. The values of magnetostriction shown in Figs. 2(a) and 2(b) are discussed further in Sec. IV.

Next we consider a $\phi 7 \times 0.1 \mathrm{~mm}$ thin disk with a demagnetizing factor $\mathcal{N} \approx 0.97$, which shows a quite different domain pattern displayed in Fig. 4. Most of the surface is covered by long parallel domains orientated along [100] or [010] that are as wide as $200 \mu \mathrm{m}$ [Figs. 4(a) and 4(c)], but there are some areas of narrow domains [Figs. 4(b) and 4(d)], which are only about $6 \mu \mathrm{m}$ wide. The long wide barlike domains, which are actually $180^{\circ}$ domains that are magnetized along the surface with parallel easy axes, are expected to carry on through the $100 \mu \mathrm{m}$ thickness of the crystal. In order to study the domain structure during the magnetization process, MOKE images were recorded as a function of in-plane magnetic field (Fig. 5). Two representative spots "a" and "c" are chosen,

(a)

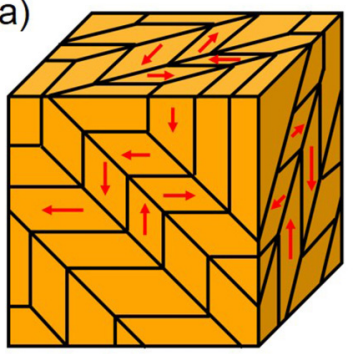

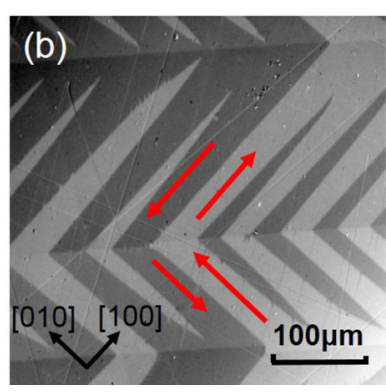

FIG. 3. Schematic of the twinlike domain structure of isotropic sample in a stress-free demagnetized state.
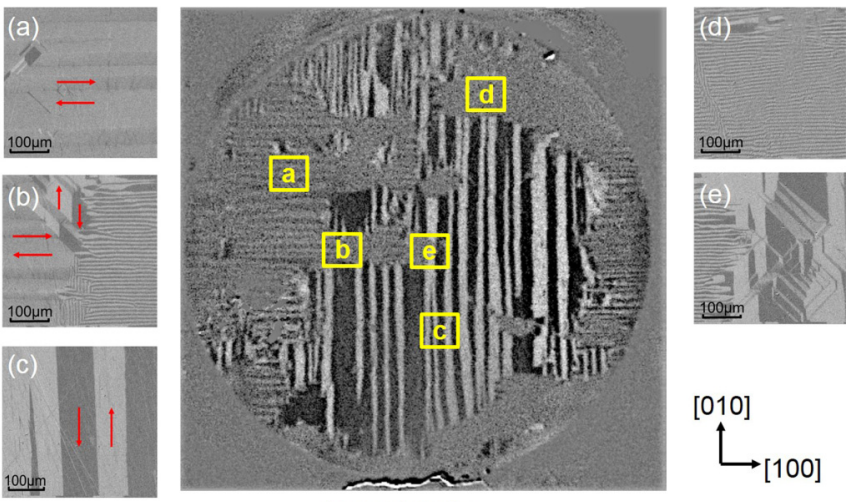

(f)

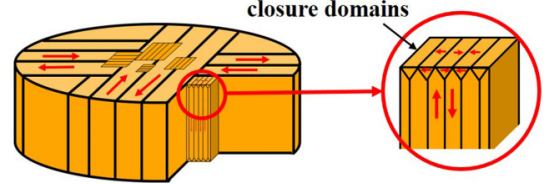

[010]

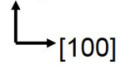

FIG. 4. Kerr microscopy image of a $\phi 7 \times 0.1 \mathrm{~mm}$ sample. (a) to (e) Images of spots a to e, respectively. There are big in-plane bar domains and narrow closure domains along [100] and [010]. The schematic domain structure is shown in (f), where the bar domains continue through the sample thickness, but the narrow domains seen in (b), (d), and (e) are interpreted as closure domains.

where domains were initially perpendicular and parallel to the applied field direction. The full MOKE loops, based on the reflected intensities, are presented in Fig. 5(a), and the

(a)
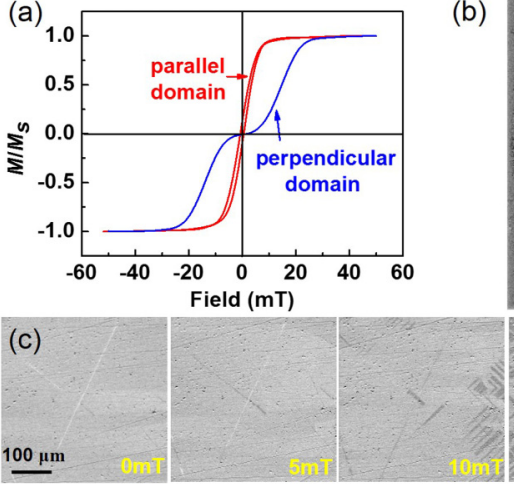

(d)

(e)

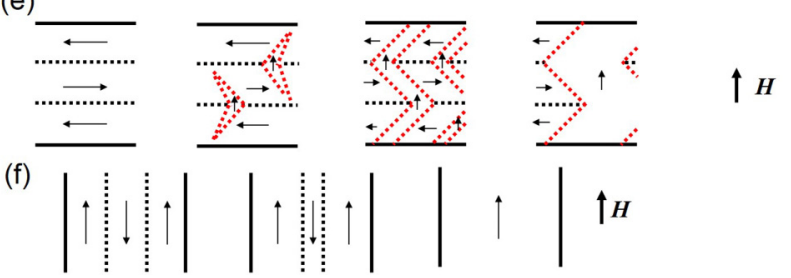

FIG. 5. MOKE curves on the $\phi 7 \times 0.1 \mathrm{~mm}$ sample. (a) $M-H$ curves deduced from the domain areas of two regions a and $\mathrm{c}$ where the field is applied perpendicular and parallel to the bar domains. (c) and (d) are images showing the domain reorientation and domain wall motion a and $\mathrm{c}$ in regions, respectively. The two processes are illustrated schematically in (e) and (f). 

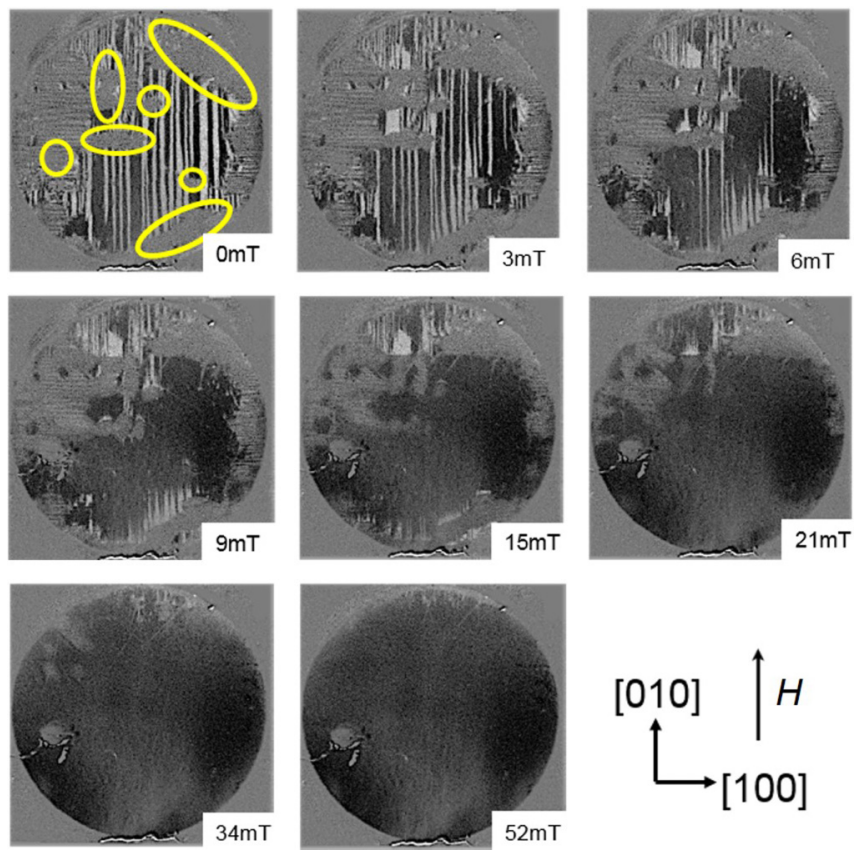

FIG. 6. In situ Kerr microscopy images with a magnetic field along [010]. The area of narrow closure domains are marked by yellow ovals.

evolution of the domain structure is illustrated in Figs. 5(c) and 5(d). For the big barlike domains parallel to the field, the $180^{\circ}$ domain walls start to move almost at once in a very small field $(<1.7 \mathrm{mT})$ and quickly reach saturation in less than $10 \mathrm{mT}$ [Fig. 5(d)]. However, for the bar transverse domains, a shallow initial slope is observed in the $M-H$ curve in Fig. 5(a), consistent with the observation in Fig. 5(c) of a threshold for nucleation of longitudinal domains. More than $20 \mathrm{mT}$ is required for saturation. Similar phenomenon of in-plane rotation of magnetic bar domains is observed in Fe-Ga thin films [20].

The narrow domains that occupy about a quarter of the surface [Fig. 4(d)] look as if they are parallel to the surface, but their behavior is quite different, as shown in Figs. 6 and 7, where a variable field is applied in the [100] or [110] direction. These narrow domains, marked out by yellow ovals in Figs. 6 and 7, remain unchanged up to $9 \mathrm{mT}$, when they gradually disappear. The big parallel domains need less than $6 \mathrm{mT}$ to reorientate. We infer that the narrow domains are closure domains at the surface with perpendicular domains just below the surface [Fig. 4(f)]. The narrow domains are closure domains of internal basic domains that are magnetized along the perpendicular easy axis, forming a "V-line" pattern at the surface [2].

Since different areas in the $\phi 7 \times 0.1 \mathrm{~mm}$ disk show different domain patterns, magnetostriction measured in the middle (an area with both narrow domains and wide bar domains along [010]) and at the edge (an area with only wide bar domains) is quite different, as shown in Fig. 8. The disk is too thin to measure magnetostriction along [001] directly with a strain gauge. Magnetostriction measured in the middle with strain gauges along [100] [Fig. 8(c)] and [010] [Fig. 8(d)] is quite different for both parallel and perpendicular magnetic field, but
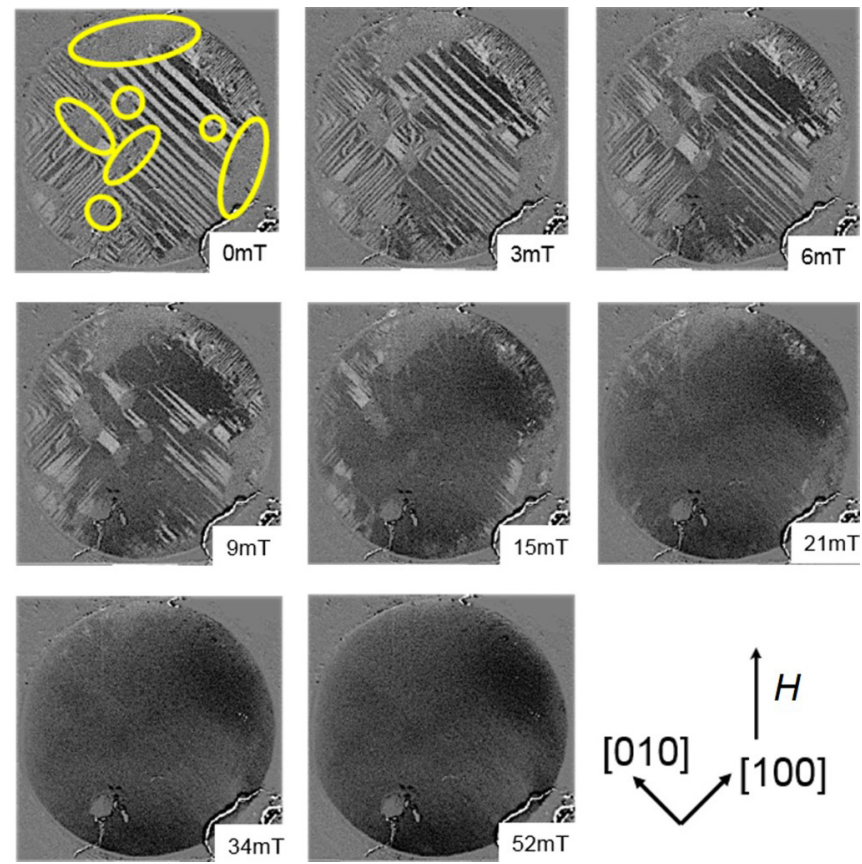

FIG. 7. In situ Kerr microscopy with a magnetic field along [110]. The area of narrow closure domains are marked by yellow ovals.

the intrinsic tetragonal and trigonal magnetostriction constants $(3 / 2) \lambda_{100}$ (deduced from $\lambda_{\|}-\lambda_{\perp}$ ) and $3 \lambda_{111}$ (deduced from $\left.\lambda_{[110]}-\lambda_{[1-10]}\right)$ remain consistent at 210 and $24 \mathrm{ppm}$, respectively, regardless of where on the crystal they are measured. The difference in values compared to the $\phi 5 \times 5 \mathrm{~mm}$ crystal is attributed to a different cooling rate during single crystal growth, the width of the domains relative to the width and position of the strain gauges and the uniformity of the initial state. The $\phi 7 \times 0.1 \mathrm{~mm}$ disk was a slice of a long rod. The average magnetostriction $\lambda_{[110]}$ in the middle is about $40 \mathrm{ppm}$. But at the edge, $\lambda_{[110]}$ is close to zero. In the Discussion we will show how these magnetostriction values can be used to deduce the fraction of domains along the three $\langle 100\rangle$ directions in the unmagnetized state.

We have measured magnetostriction on nine more samples of different shapes in order to evaluate the percentage of domains that are parallel or perpendicular to the surface. One example is a $\phi 5 \times 0.88 \mathrm{~mm} \mathrm{Fe}_{83} \mathrm{Ga}_{17}$ crystal measured with strain gauges in-plane and by capacitance dilatometry in the perpendicular direction. Data are shown in Fig. 9. This sample has a demagnetizing factor $\mathcal{N}=0.72, \lambda_{\|}$is $250 \mathrm{ppm}$, while the in-plane $\lambda_{\perp}$ is $-56 \mathrm{ppm}$ and the transverse $\lambda_{\perp}$ is $-176 \mathrm{ppm}$. The value of $(3 / 2) \lambda_{100}$ deduced from the angular variation at saturation [Fig. 9(b)] is $306 \mathrm{ppm}$. Magnetostriction data on this and all the other samples are gathered in Table I. Our most complete set of data is for the $10.6 \times 10.6 \times 2.4 \mathrm{~mm}$ square sample that has been either slowly cooled or quenched from $760^{\circ} \mathrm{C}$, where the field is applied and magnetostriction measured in up to five different directions.

We show a set of representative magnetization curves in Fig. 10. Figure 10(a) shows three curves measured on a $\phi$ $5 \times 1.9 \mathrm{~mm}$ disk $(\mathcal{N}=0.54)$ cut so that the face is a $(1-10)$ plane containing [110], [111], and [001] directions. The three curves are very similar, and the magnetizations saturates in 
(a)
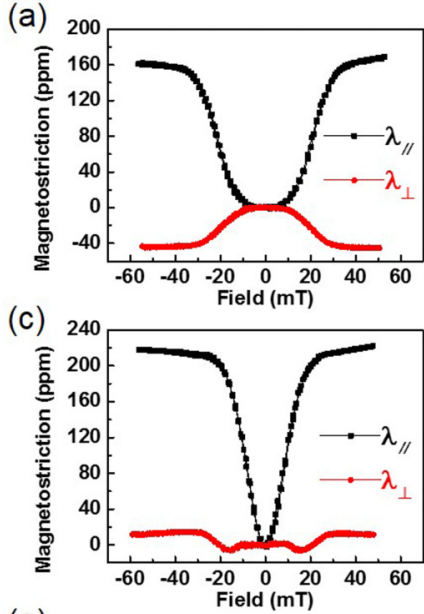

(e)
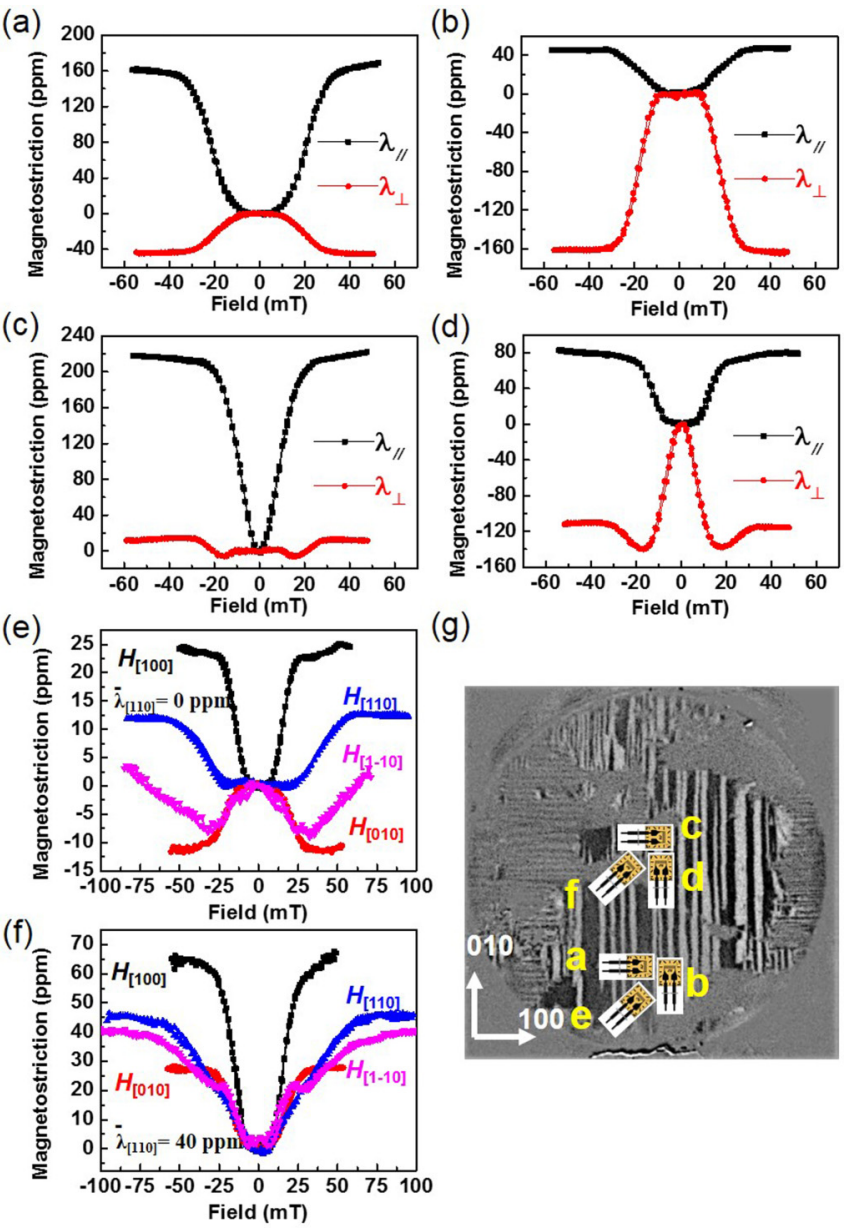

(g)

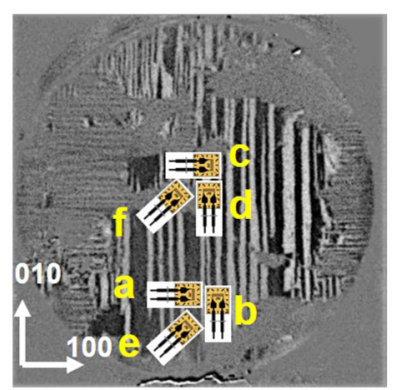

FIG. 8. Magnetostriction in different areas of the $\phi 7 \times 0.1 \mathrm{~mm}$ sample. (a)-(d) Measured with strain gauges in [100] or [010] directions in the areas a to $\mathrm{d}$ in $(\mathrm{g})$, with the field parallel or perpendicular to the measuring direction. (e) and (f) $\lambda_{[110]}$ in areas e and $\mathrm{f}$.

a field of $\mu_{0} H=0.35 \mathrm{~T}\left(280 \mathrm{kAm}^{-1}\right)$, which is close to the value of $319 \mathrm{kA} \mathrm{m}^{-1}$ expected from the magnetization $(M=$ $1.38 \mathrm{MA} \mathrm{m}^{-1}$ ) and the in-plane demagnetization factor $\mathcal{N}^{\prime}=$ $(1-\mathcal{N}) / 2=0.23$. In-plane magnetization curves are shown for other crystals with $\mathcal{N}^{\prime}$ ranging from 0.14 to 0.33 . Note that the saturation is never quite complete at $H=\mathcal{N}^{\prime} M$. (a)

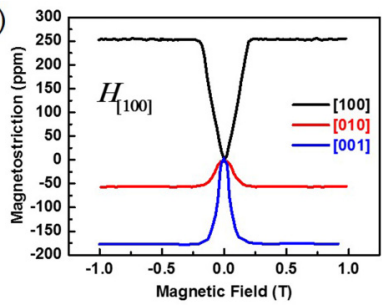

(b)

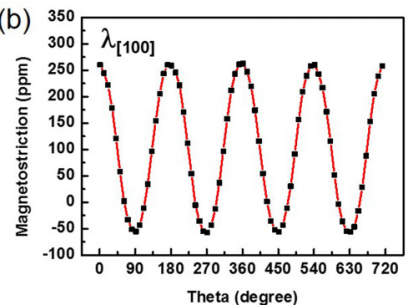

FIG. 9. Magnetostriction of a $\phi 5 \times 0.88 \mathrm{~mm} \mathrm{Fe}{ }_{83} \mathrm{Ga}_{17}$ single crystal. (a) Magnetostriction in three orthogonal directions [100], [010], and [001] when the magnetic field $H$ is applied along [100]. (b) Saturation magnetostriction measured along [100] when an applied field of $1 \mathrm{~T}$ is rotated in the plane of the disk.
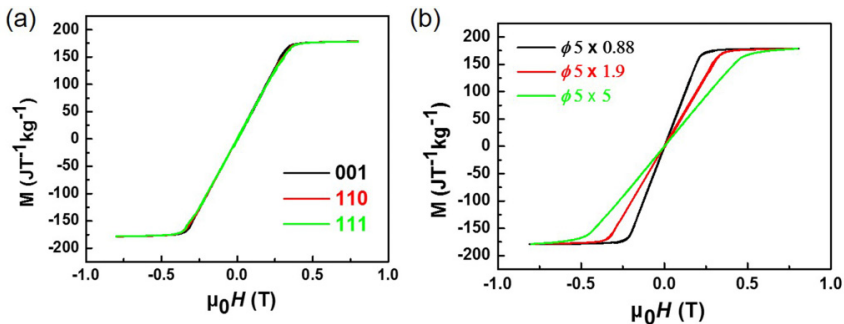

FIG. 10. Magnetization curves of $\mathrm{Fe}_{83} \mathrm{Ga}_{17}$ single crystals with different shapes and measuring directions. (a) Magnetization curves of a $\phi 5 \times 1.9 \mathrm{~mm}$ disk measured in [110], [111], and [001] directions. (b) In-plane [100] magnetization curves for three crystals with different in-plane demagnetizing factors $\mathcal{N}^{\prime}$. The samples are as-grown.

Finally, we measured Possion's ratio for a $5 \times 5 \times 5$ cubic crystal with (100), (010), and (001) faces and another $5 \times 5 \times 5$ cubic crystal with (110), (1-10), and (001) faces. Results

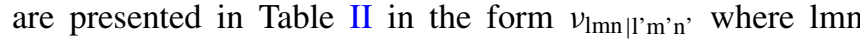
is the stress direction and Possion's ratio is the strain ratio $v=-\varepsilon_{l^{\prime} \mathrm{m}^{\prime} \mathrm{n}^{\prime}} / \varepsilon_{\mathrm{Imn}}$. The [110] direction is an auxetic (negative v) direction in bec crystals [35] and the $v_{110 \mid 1-10}$ value is -0.86 , in agreement with other reports [36]. The [001] strain when stress is applied in an auxetic direction is particularly large. Note that the crystal volume is conserved under strain for $\langle 100\rangle$ directions $(v \approx 1 / 2)$, but not for $\langle 110\rangle$ directions.

\section{DISCUSSION}

\section{A. Domain distributions}

First we explain how we deduce the volume fraction of domains in the $\langle 100\rangle$ easy directions. The standard expression for the saturation magnetostriction of a crystal with cubic symmetry is [11]

$$
\begin{aligned}
\frac{\Delta 1}{l}= & \frac{3}{2} \lambda_{100}\left(\alpha_{x}{ }^{2} \beta_{x}{ }^{2}+\alpha_{y}{ }^{2} \beta_{y}{ }^{2}+\alpha_{z}{ }^{2} \beta_{z}{ }^{2}-\frac{1}{3}\right) \\
& +3 \lambda_{111}\left(\alpha_{x} \alpha_{y} \beta_{x} \beta_{y}+\alpha_{y} \alpha_{z} \beta_{y} \beta_{z}+\alpha_{z} \alpha_{x} \beta_{z} \beta_{x}\right),
\end{aligned}
$$

where $\lambda_{100}$ and $\lambda_{111}$ are the two intrinsic saturation magnetostriction constants, and $\alpha_{i}$ and $\beta_{i}$ are the direction cosines of the magnetization direction and the measurement direction, respectively. We make no a priori assumption about the domain

TABLE II. Possion's ratios for an $\mathrm{Fe}_{83} \mathrm{Ga}_{17}$ as-grown single crystal. The figure [35] illustrates the auxetic effect in a bcc crystal stressed in a [110] direction. It expands along [110], but also along [1-10], while it contracts along [001].

\begin{tabular}{ccc}
\hline \hline $\begin{array}{c}\text { Direction } \\
\text { Stress/strain }\end{array}$ & $\begin{array}{c}\text { Poisson's ratio } \\
-\varepsilon_{l^{\prime} \mathrm{m}^{\prime} \mathrm{n}} / \varepsilon_{\mathrm{Imn}}\end{array}$ \\
\hline $100 \mid 010$ & 0.47 \\
$100 \mid 001$ & 0.47 \\
$110 \mid 1-10$ & -0.86 \\
$110 \mid 001$ & 1.05
\end{tabular}


structure in the initial demagnetized state, but denote it by $\left(n_{x}, n_{y}, n_{z}\right)$ where $n_{x}, n_{y}$, and $n_{z}$ are the volume fractions of domains oriented along [100], [010], or [001] with the constraint $n_{x}+n_{y}+n_{z}=1$. Inserting the appropriate direction cosines for the three domains and summing over them, the predicted saturation magnetostriction based on Eq. (1) is represented by the array

$$
\frac{\Delta l}{l}=\frac{3}{2} \lambda_{100}\left[\begin{array}{ccc}
n_{y}+n_{z} & -n_{y} & -n_{z} \\
-n_{x} & n_{x}+n_{z} & -n_{z} \\
-n_{x} & -n_{y} & n_{x}+n_{y}
\end{array}\right],
$$

where the rows correspond to the [100], [010], and [001] field directions and the columns correspond to the [100], [010], and [001] measurement directions for magnetostriction. The array is used as a table with the appropriate element chosen from cross referencing the applied field direction, defining the row, with the measurement direction, defining the column. The rows in Eq. (2) correspond to the [100], [010], and [001] field directions and the columns correspond to the [100], [010], and [001] measurement directions for magnetostriction. Similarly

$$
\begin{aligned}
\frac{\Delta l}{l}= & \frac{3}{2} \lambda_{100}\left[\begin{array}{ccc}
1 / 2 n_{z} & 1 / 2 n_{z} & -n_{z} \\
1 / 2 n_{z} & 1 / 2 n_{z} & -n_{z} \\
-1 / 2\left(n_{x}+n_{y}\right) & -1 / 2\left(n_{x}+n_{y}\right) & n_{x}+n_{y}
\end{array}\right] \\
& +3 \lambda_{111}\left[\begin{array}{ccc}
1 / 4\left(n_{x}+n_{y}\right) & -1 / 4\left(n_{x}+n_{y}\right) & 0 \\
-1 / 4\left(n_{x}+n_{y}\right) & 1 / 4\left(n_{x}+n_{y}\right) & 0 \\
0 & 0 & 0
\end{array}\right],
\end{aligned}
$$

where the corresponding orthogonal directions are now [110], [1-10], and [001]. Note that the elements in (2) and (3) are all obtained from (1) as the differences between the magnetostriction in the initial multidomain state and the saturated state.

Let us take the first element in the array of (2) as an example: For an initial $\pm x$ domain, $\alpha_{x}= \pm 1, \alpha_{y}=0, \alpha_{z}=0$. If we measure along the $x$ direction, then $\beta_{x}=1, \beta_{y}=0, \beta_{z}=0$. By putting these numbers into Eq. (1), $\frac{\Delta 1}{l}=\lambda_{100}$, but there is no difference in strain between the initial and final states when we flip the $\alpha_{x}=-1$ domains, it remains $\frac{\Delta 1}{l}=\lambda_{100}$. The sample shape does not change when we compare before and after the magnetization process of the $n_{x}$ domains, so they do not contribute to this element of the magnetostriction. However, for an initial $y$ domain, $\frac{\Delta 1}{l}=-\frac{1}{2} \lambda_{100}$, as $\alpha_{x}=$ $0, \alpha_{y}= \pm 1, \alpha_{z}=0$, and $\beta_{x}=1, \beta_{y}=0, \beta_{z}=0$ when we are measuring along $x$. When a field is applied along $x$, the magnetization rotates to $\alpha_{x}=1, \alpha_{y}=0, \alpha_{z}=0$, and $\frac{\Delta 1}{l}=$ $\lambda_{100}$. The net magnetostriction contributed by $y$ domain is $\lambda_{100}-\left(-\frac{1}{2} \lambda_{100}\right)=\frac{3}{2} \lambda_{100}$. The same is true for an initial $z$ domain, $\frac{\Delta l}{l}=\frac{3}{2} \lambda_{100}$. All together, the magnetostriction in the first element of the array is $0 \times n_{x}+\frac{3}{2} \lambda_{100} \times n_{y}+\frac{3}{2} \lambda_{100} \times$ $n_{z}=\frac{3}{2} \lambda_{100}\left(n_{y}+n_{z}\right)$, which is the number of the first line and the first column of the array in Eq. (2). Similarly for all the other elements.

Three independent measurements of saturation magnetostriction are needed to determine $\lambda_{100}$ and $\left(n_{x}, n_{y}, n_{z}\right)$, and four independent measurements are needed to determine $\lambda_{100}, \lambda_{111}$, and $\left(n_{x}, n_{y}, n_{z}\right)$. The data in Table I overdetermine the measurement in every case, especially for the $10.6 \times$ $10.6 \times 2.4 \mathrm{~mm}^{3}$ sample, where there are 17 independent measurements. We can therefore use our data to evaluate errors in the measurements, related for example to the width of the domains relative to the width and position of the strain gauges and the uniformity of the initial state, as well as to investigate any systematic discrepancies from the predictions of Eqs. (1)-(3), which are based on volume conserving "Joulian magnetostriction" [32]. Note that the sum of the elements in any row of Eq. (2) or (3) is zero, and that it is possible to find combinations of elements in the columns that are independent of the distribution of domains in the initial state because of the constraint $n_{x}+n_{y}+n_{z}=1$.

First we revisit the $\phi 5 \times 5 \mathrm{~mm}$ crystal of Figs $1-3$. From the magnetostriction constants $\lambda_{100}=187$ and $\lambda_{111}=9 \mathrm{ppm}$ determined at saturation by rotating the $1 \mathrm{~T}$ field, and an isotropic distribution $(1 / 3,1 / 3,1 / 3)$ of domains in the initial state, we deduce saturation magnetostriction values in the [100], [010], and [001] directions for $H_{[100]}$ of $187,-94$, and $-94 \mathrm{ppm}$, and values in the [110], [1-10], and [001] directions for $H_{[110]}$ of 51, 42, and $-94 \mathrm{ppm}$. These differ slightly from the measurements for this crystal reported in Table I, but the agreement cannot be improved by a nonisotropic initial domain configuration, as it will not influence the row sums in Eq. (2) or (3).

There are some possible nonvolume-conserving contributions to the strain, which may contribute to the small discrepancies from the Joulian (volume-conserving) theory. One is the isotropic spontaneous volume magnetostriction [11], which is slightly field dependent in ferromagnetic metals. Another is the form effect [11]. Any magnetized sample experiences a Maxwell stress in a uniform field [3]. The stress is easily calculated from the surface magnetic "charge" density. The stress on the two faces is $\pm B M$, where $B$ and $M$ are flux density and magnetization, respectively. For a cubic sample, the saturation field $B=0.5 \mathrm{~T}, M=1.4 \mathrm{MA} / \mathrm{m}$, this stress is $0.7 \mathrm{MN} / \mathrm{m}^{2}$ (0.7 MPa). Taking Young's modulus $E=64 \mathrm{GPa}$ [37], we have an $11 \mathrm{ppm}$ strain, which looks as if it were magnetostriction, but it only conserves volume if Poisson's ratio is $1 / 2$. This response to an imposed external stress that does not necessarily conserve volume is a phenomenon that is distinct from the intrinsic linear (directional) magnetostriction of a magnetically ordered material. In other metals like $\mathrm{Fe}$ where Young's modulus is bigger, the effect is reduced.

Nevertheless, any non-Joulian contribution to the magnetostriction of $\mathrm{Fe}_{83} \mathrm{Ga}_{17}$ must be very small. Applying the Joulian test $\Sigma_{i=1}{ }^{3} \lambda_{i}$ to all 27 data sets for which we have complete row sums, we find that the mean value of the row sum is just $6 \mathrm{ppm}$, with a standard deviation of $\pm 8 \mathrm{ppm}$. By contrast, the mean row sum of the moduli $\Sigma_{i=1}{ }^{3}\left|\lambda_{i}\right|$ is $280 \mathrm{ppm}$, so we conclude that the data would be consistent with a non-Joulian contribution that is about $2 \%$ of the total effect, but that random errors related to positioning of the strain gauges or uncertainty in deciding the saturation level from data such as are shown in Fig. 2 or 8 can be at least as large.

For the $\phi 7 \times 0.1 \mathrm{~mm}$ crystal of Figs. $4-8$, the fitted values of the domain distribution and magnetostriction constants for the central area of Fig. 8 are $(0,57,43) \%, \frac{3}{2} \lambda_{100}=202 \mathrm{ppm}$, $3 \lambda_{111}=18 \mathrm{ppm}$, whereas for the edge area the fitted values are $(21,79,0) \%, \frac{3}{2} \lambda_{100}=202 \mathrm{ppm}, 3 \lambda_{111}=20 \mathrm{ppm}$. Note that the magnetostriction constants are identical for parts of a crystal with quite different domain orientations in the initial state, but 
TABLE III. Magnetostrictive effects in $\mathrm{Fe}_{83} \mathrm{Ga}_{17}$.

\begin{tabular}{lccc}
\hline \hline Strain type & $\begin{array}{c}\text { Value } \\
(\mathrm{ppm})\end{array}$ & JM/NJM & $\begin{array}{c}\text { Isotropic/ } \\
\text { anisotropic }\end{array}$ \\
\hline Linear saturation MS $\lambda_{100}$ & 170 & Joulian & Anisotropic \\
Forced volume MS in 1 T & 14 & Non-Joulian & Isotropic \\
Form effect in 1 T [110] & 8 & Non-Joulian & Anisotropic \\
Auxetic effect [110] (unsaturated) & 8 & Non-Joulian & Anisotropic \\
$\Delta$ E effect [100] (unsaturated) & 2 & Non-Joulian & Anisotropic \\
\hline \hline
\end{tabular}

the values of $\lambda_{100}$ vary from 137 to $199 \mathrm{ppm}$ and values of $\lambda_{111}$ vary from 6 to $18 \mathrm{ppm}$, depending on heat treatment.

Next we justify the interpretation of the narrow domains (Fig. 4) as closure domains, which form above the perpendicular domains to eliminate the stray field. Following Kittel [1], minimization of the sum of domain wall energy and magnetoelastic energy due to the strain misfit at the $90^{\circ}$ domain walls gives the closure domain width $D$ as

$$
D=\frac{2}{\lambda_{100}} \sqrt{\frac{\gamma_{w} L}{C_{11}}},
$$

where $L$ is the thickness of the crystal, $C_{11}$ is an elastic constant which is $200 \mathrm{GPa}$ [38], and $\gamma_{w}$ is the $90^{\circ}$ domain wall energy. Relevant experimental data for $\mathrm{Fe}_{83} \mathrm{Ga}_{17}$ are given in Table III. The width of the closure domain according to Eq. (4) is $6 \mu \mathrm{m}$ for the $\phi 7 \times 0.1 \mathrm{~mm}$ sample, which agrees well with the experimental result of 5-6 $\mu \mathrm{m}$.

Figure 11 summarizes the relationship between sample shape, longitudinal magnetostriction $\lambda_{\|}$, and domain proportions. Shape is parametrized in terms of the in-plane demagnetizing factor $\mathcal{N}^{\prime}$. The domain distribution in the initial state determines the magnetostriction curve. With decreasing $\mathcal{N}^{\prime}$ (thinner samples), $\lambda_{\|}$may increase from $\lambda_{100}$ for isotropic samples with $\mathcal{N}^{\prime}=0.33$ to $(3 / 2) \lambda_{100}$ when $\mathcal{N}^{\prime} \approx 0$, indicating that shape anisotropy induces magnetostrictive anisotropy $\left(\lambda_{\|}\right.$ no longer equals $-2 \lambda_{\perp}$ ). The greatest possible magnetostrictive strain is realized when the initial domain structure has all domains magnetized perpendicular to the field direction. When the field is applied along $x$, the fraction of such domains $n_{y}+$ $n_{z}$ increases from $67 \%$ for the isotropic $\phi 5 \times 5 \mathrm{~mm}$ sample to $100 \%$ at the middle of the $\phi 7 \times 0.1 \mathrm{~mm}$ sample, due to the absence of the $x$-bar domains $\left(n_{x}=0\right)$. In Fig. $11, \lambda_{\|} / \lambda_{100}$ and $n_{y}+n_{z}$ follow the same trend. The values of $\left(n_{x}, n_{y}, n_{z}\right)$ vary
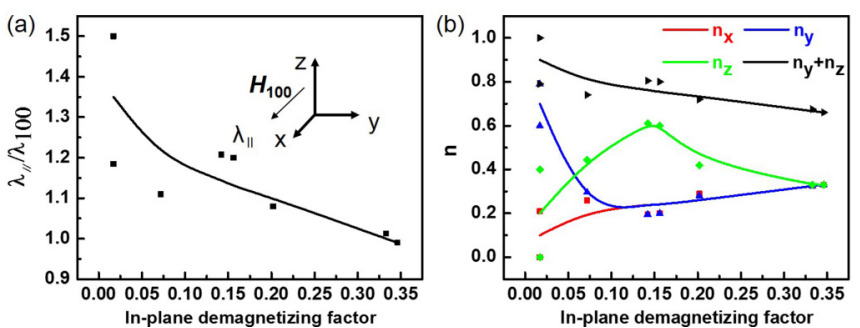

FIG. 11. Shape effects. The relationship between the in-plane demagnetizing factor $\mathcal{N}^{\prime}$ and (a) the longitude magnetostriction with field along [100] and (b) the proportions of domains in the $\langle 001\rangle$ directions. The crystals are all as-grown. (a)

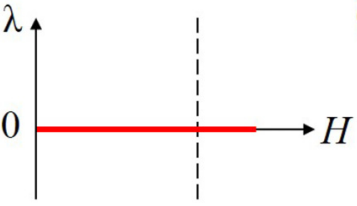

$>180^{\circ}$ domain wall motion

$>90^{\circ}$ domain reorientation $\lambda \perp \boldsymbol{M}$

$>\lambda_{[110]}$ when $\boldsymbol{H}$ and $\boldsymbol{M}$ in (001)

(c)

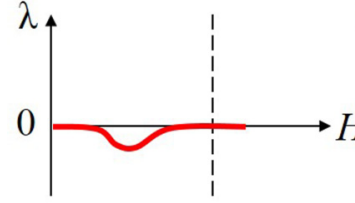

$>\boldsymbol{M}$ rotates in (001) through [110], $\lambda_{[001]}$, auxetic effect (b)

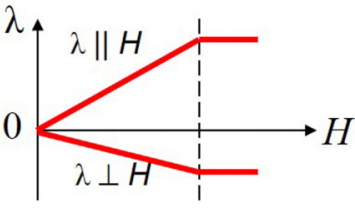

$90^{\circ}$ domain wall motion $(\lambda$ in-plane)

(d)

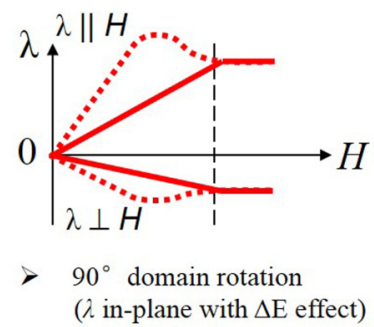

FIG. 12. Sketches of magnetostriction curves arising from different magnetization process, including the auxetic effect and the $\Delta E$ effect.

greatly among the 12 samples tested in Table I. The value of $n_{z}$ is greatest for the samples with $\mathcal{N}^{\prime} \approx 0.15$, but crystal shape and thickness both have an influence. Heat treatment can have a strong influence on the domain structure, and consequently on the magnetostriction [39], but the variation is not systematic.

\section{B. Magnetization process}

The shape of the magnetostriction curves reveals the magnetization process, which we discuss here for bcc crystals with $\langle 100\rangle$ easy axes. The elementary processes of magnetization rotation and domain wall motion have characteristic signatures. Transverse $180^{\circ}$ domain wall motion in a field parallel to the domain magnetization directions, seen for example in Fig. 5(d), does not change the magnetostriction, nor does a process of domain reorientation whether continuous or via $90^{\circ}$ domain wall motion in a plane perpendicular to the measurement direction.

Continuous magnetization rotation towards the measurement directions gives a continuous increase of magnetostriction up to saturation, whereas it gives a continuous decrease when the rotation is away from the measurement direction. A similar effect is found in $90^{\circ}$ domain wall motion in a plane containing the measurement direction. These elementary processes are illustrated in Figs. 12(a) and 12(b).

Two special effects can be associated with magnetization rotation (but not $90^{\circ}$ domain wall motion), which are not taken into account by Eq. (1). The first of these is the auxetic effect [35,36] presented in Table III. This arises when the moment passes through a [110] direction during the magnetization process. At this point, the deformation along [001] is exceptionally large, and this is manifest as a bump, which is eliminated when the magnetization finally reaches the [100] direction [Fig. 12(c)].

The second special effect relates to the $\Delta E$ effect, which is a softening of Young's modulus $E$ due to rotation of magnetostrictive domains during the magnetization process 

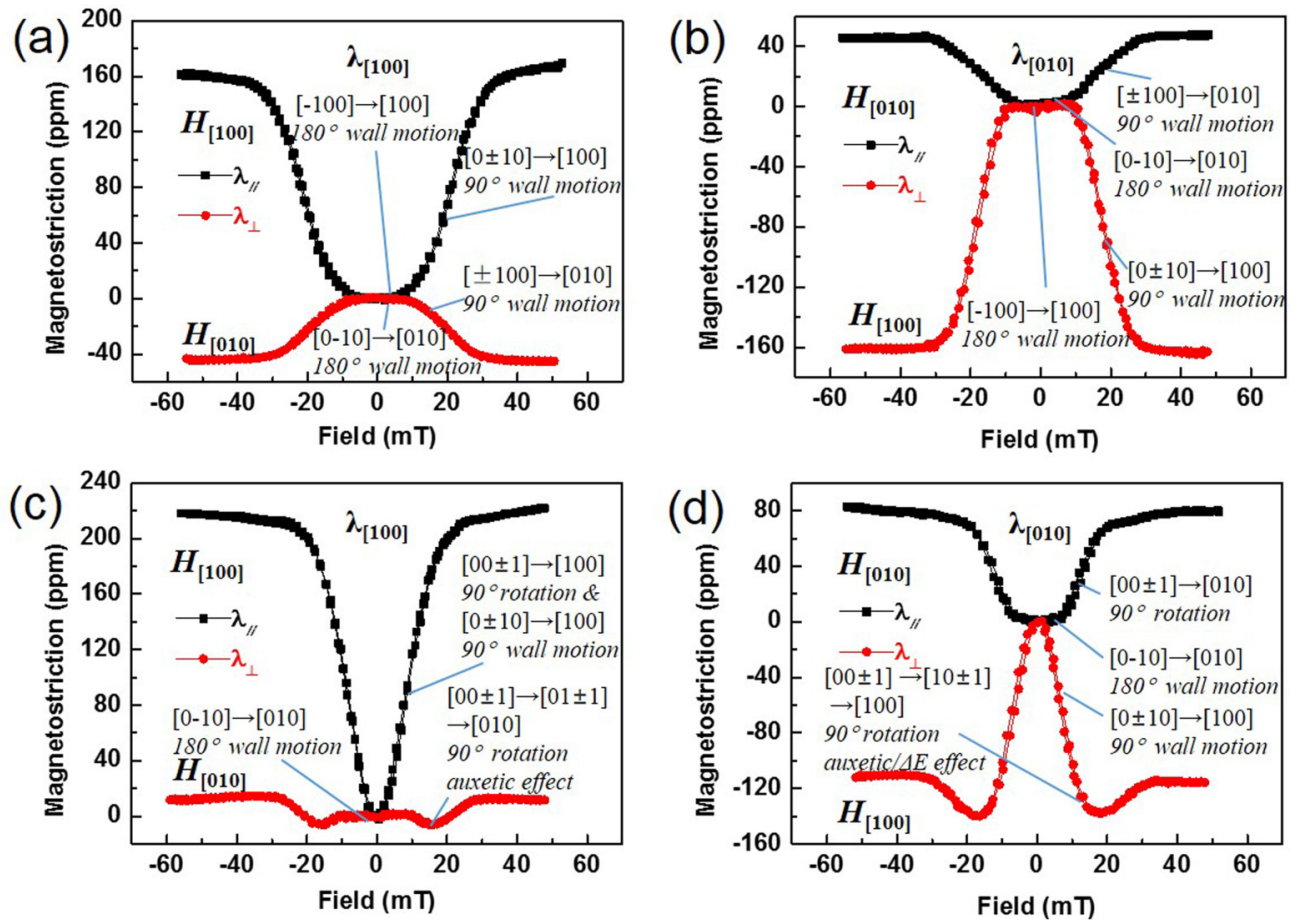

FIG. 13. Magnetization process in the $\phi 7 \times 0.1 \mathrm{~mm}$ sample. Data from Fig. 8 labeled to indicate the magnetization processes.

[3]. The decrease of $E$ leads to an increase in strain, which then disappears at saturation [Fig. 12(d). The effect is similar to applied stress, which favors domain orientation perpendicular to the stress direction. Data of [40] suggest the $\Delta E$ effect in $\mathrm{Fe}_{82} \mathrm{Ga}_{9} \mathrm{Al}_{9}$ may be of order $25 \%$. Comparison of different magnetostrictive (MS) effects in $\mathrm{Fe}_{83} \mathrm{Ga}_{17}$ alloys is shown in Table III. The effects of internally generated magnetostrictive strain are expected to be purely Joulian, on account of the isotropic character of the exchange overlap integrals, which leads to volume conservation. Any corrections due to anisotropic exchange are smaller by approximately two orders of magnitude (in energy) for close-packed metallic systems with low spin-orbit coupling [41].

Finally we summarize the magnetization process in the $\phi 7 \times 0.1 \mathrm{~mm}$ sample in terms of these mechanisms. The data are reproduced in Fig. 13, and the labels mark what is happening to the domains at each stage in the magnetostriction curve.

\section{CONCLUSIONS}

We have presented a method for studying quantitatively the domain structure and magnetization process in bulk ferromagnetic materials, illustrating it with data on highly magnetostrictive bcc $\mathrm{Fe}_{83} \mathrm{Ga}_{17}$ crystals. The combination of Kerr microscopy and magnetostriction measurements shows that sample shape and heat treatment greatly influence the initial domain structure. Only in isometric bulk samples are the domains equally distributed along all three easy axes. The distribution $\left(n_{x}, n_{y}, n_{z}\right)$ can be deduced in a general case from the saturation magnetostriction measured in different crystallographic directions, with different orientations of the applied magnetic field. Saturation magnetostriction for all the $\mathrm{Fe}_{83} \mathrm{Ga}_{17}$ crystals is accurately represented by Eqs. (2) and (3), which are derived from Eq. (1), regardless of crystal shape or heat treatment. We find that the saturation magnetostriction in $\mathrm{Fe}_{83} \mathrm{Ga}_{17}$ crystals is volume conserving within about $2 \%$ despite a recent claim to the contrary that could not be repeated on epitaxial films [42], which we discussed in [32].

Useful information can also be gleaned about the magnetization process from the shape of the magnetostriction curve. In particular, domain rotation process that pass through an auxetic $\langle 110\rangle$ direction, or give rise to the $\Delta E$ effect have characteristic signatures that have been observed but not previously explained in many magnetostriction measurement on Fe-Ga crystals $[12,15,40,43-45]$. These processes do not necessarily conserve the sample volume, and they merit further investigation.

\section{ACKNOWLEDGMENTS}

We would like to thank the Dr. Jun Lu for the help with measurements of the magnetization curves. We are grateful to Prof. Plamen Stamenov for help and advice on the construction of the capacitance device and to Dr. Bodhan Kundys for some of the perpendicular magnetostriction measurments. C.B.J. was supported by the National Natural Science Foundation of China (NSFC) under Grant No. 51331001. J.M.D.C. was supported as a Thousand Talent Professor of Beihang University, and the work was partly supported by Science Foundation Ireland under contact 13/ERC/I2561.

\section{APPENDIX: DETAILS OF MAGNETOSTRICTION MEASUREMENT METHODS}

For measurements of the perpendicular magnetostriction using the capacitance method, the polished sample surface and a 50-nm-thick Ti/Au layer evaporated on a sapphire single crystal plate constitute the measurement capacitor. The sapphire is fixed to the brass support 
(a)

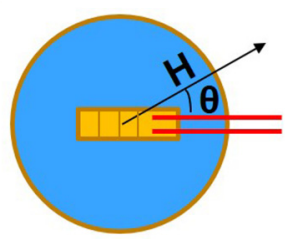

(c)

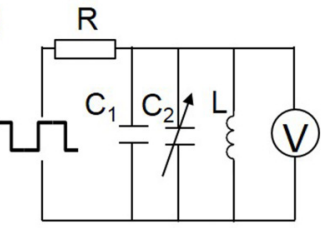

(b)

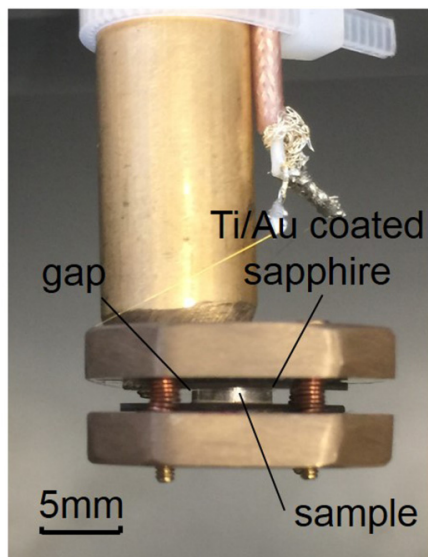

FIG. 14. Details of magnetostriction measurement methods. (a) Standard strain gauge measurement, with the magnetic field rotated in the plane perpendicular to the axis of the magnet bore without moving the sample. (b) Image of the capacitance dilatometer. (c) Circuit diagram for the measurement.

structure using superglue, and the base of the sample is mounted using silver paint. The parallelicity and the capacitor gap are controlled to better than $5 \mu \mathrm{m}$ using a set of three M1.2 brass screws and beryllium bronze springs. Apart from the sample and the sapphire plates, only brass is used in the construction. During measurements, the additional calibration capacitance of the decade capacitance box is set to $C_{2}=0$ and the phase shift of the first harmonic is measured versus applied magnetic field using a DSP lock-in amplifier with square wave excitation with frequency of approximately $100 \mathrm{kHz}$ (Fig. 14). The frequency of the signal generator is defined to 1 part in $10^{8}$. The set distance between the capacitor plates is estimated using capacitance difference using as reference a dilatometer gap distance of about $1 \mathrm{~mm}$, in order to render $C_{1}$ negligible and measure the absolute phase shift corresponding to the set capacitance of the dilatometer. Finally, a phase-shift-tocapacitance calibration is performed, using discrete changes in the $C_{2}$ decade capacitor. (The change in $C_{1}$ is of order 1 $\mathrm{pF}$, and the capacitances in the decade box are $1-100 \mathrm{pF}$.) The experimental uncertainty in this capacitance dilatometry measurement is estimated as $\pm 30 \mathrm{ppm}$.
[1] C. Kittel, Physical theory of ferromagnetic domains, Rev. Mod. Phys. 21, 541 (1949).

[2] A. Hubert and R. Schäfer, Magnetic Domains (Springer, Berlin, 1998).

[3] J. M. D. Coey, Magnetism and Magnetic Materials (Cambridge University Press, Cambridge, 2010).

[4] Y. Zhu, Modern Techniques for Characterizing Magnetic Materials (Springer, New York, 2005), Chap. 11.

[5] M. R. Scheinfein, J. Unguris, M. Aeschlimann, D. T. Pierce, and R. J. Celotta, Scanning electron microscopy with polarization analysis (SEMPA) - Studies of domains, domain walls and magnetic singularities at surfaces and in thin films, J. Magn. Magn. Mater. 93, 109 (1991).

[6] J. Baruchel, X-ray and neutron topographical studies of magnetic materials, Physica B 192, 79 (1993).

[7] J. Pollmann, G. Srajer, D. Haskel, J. C. Lang, J. Maser, J. S. Jiang and S. D. Bader, Magnetic imaging of a buried SmCo layer in a spring magnet, J. Appl. Phys. 89, 7165 (2001).

[8] M. Schlenker and J. Baruchel, Neutron topography: A review, Physica B 137, 309 (1986).

[9] C. Grünzweig, C. David, O. Bunk, M. Dierolf, G. Frei, G. Kühne, R. Schäfer, S. Pofahl, H. M. R. Rønnow, and F. Pfeiffer, Bulk magnetic domain structures visualized by neutron dark-field imaging, Appl. Phys. Lett. 93, 112504 (2008).

[10] I. Manke, N. Kardjilov, R. Schäfer, A. Hilger, M. Strobl, M. Dawson, C. Grünzweig, G. Behr, M. Hentschel, C. David, A. Kupsch, A. Lange, and J. Banhart, Three-dimensional imaging of magnetic domains, Nat. Commun. 1, 125 (2008).

[11] B. D. Cullity and C. D. Graham, Introduction to Magnetic Materials, 2nd ed. (Wiley, Hoboken, NJ, 2009), Chaps. 8 and 9.

[12] H. D. Chopra and M. Wuttig, Non-Joulian magnetostriction, Nature (London) 521, 340 (2015).

[13] C. Mudivarthi, S. M. Na, R. Schaefer, M. Laver, M. Wuttig, and A. B. Flatau, Magnetic domain observations in $\mathrm{Fe}-\mathrm{Ga}$ alloys, J. Magn. Magn. Mater. 322, 2023 (2010).
[14] S. Asano, S. Fujieda, S. Hashi, K. Ishiyama, T. Fukuda, and S. Suzuki, Magnetic domain structure and magnetostriction of Fe-Ga alloy single crystal grown by the Czochralski method, IEEE Magn. Lett. 8, 6101004 (2017).

[15] G. Raghunath and A. B. Flatau, Study of magnetic domain evolution in an auxetic plane of Galfenol using Kerr microscopy, J. Appl. Phys. 117, 17E704 (2015).

[16] F. Bai, J. Li, D. Viehland, D. Wu, and T. A. Lograsso, Magnetic force microscopy investigation of domain structures in $\mathrm{Fe}-\mathrm{x}$ at.\% Ga single crystals $(12<x<25)$, J. Appl. Phys. 98, 023904 (2005).

[17] F. Bai, H. Zhang, J. Li, and D. Viehland, Magnetic force microscopy investigation of the static magnetic domain structure and domain rotation in Fe-x at.\% Ga alloys, Appl. Phys. Lett. 95, 152511 (2009)

[18] J. K. Zhou, D. D. Li, and J. G. Li, Magnetic force microscopy observation of under-cooled $\mathrm{Fe}_{81} \mathrm{Ga}_{19}$ magnetostrictive alloys, J. Phys. D: Appl. Phys. 41, 205405 (2008).

[19] H. Z. Song, Y. X. Li, K. Y. Zhao, H. R. Zeng, S. X. Hui, G. R. $\mathrm{Li}, \mathrm{Q}$. R. Yin, and G. H. Wu, Influence of stress on the mangetic domain structure in $\mathrm{Fe}_{81} \mathrm{Ga}_{19}$ alloys, J. Appl. Phys. 105, 013913 (2009).

[20] S. Fin, R. Tomasello, D. Bisero et al., In-plane rotation of magnetic stripe domains in $\mathrm{Fe}_{1-x} \mathrm{Ga}_{x}$ thin films, Phys. Rev. B 92, 224411 (2015).

[21] Q. Xing and T. A. Lograsso, Magnetic domains in magnetostrictive Fe-Ga alloys, Appl. Phys. Lett. 93, 182501 (2008).

[22] C. Mudivarthi, M. Laver, J. Cullen, A. B. Flatau, and M. Wuttig, Origin of magnetostriction in Fe-Ga, J. Appl. Phys. 107, 09A957 (2010).

[23] R. C. O’Handley, Modern Magnetic Materials (Wiley, New York, 2000).

[24] M. Abes, C. T. Koops, S. B. Hrkac, J. McCord, N. O. Urs, N. Wolff, L. Kienle, W. J. Ren, L. Bouchenoire, B. M. Murphy, and O. M. Magnussen, Domain structure and reorientation in $\mathrm{CoFe}_{2} \mathrm{O}_{4}$, Phys. Rev. B 93, 195427 (2016). 
[25] A. E. Clark, J. B. Restorff, M. Wun-Fogle, T. A. Lograsso, and D. L. Schlagel, Magnetostrictive properties of body-centered cubic Fe-Ga and Fe-Ga-Al alloys, IEEE Trans. Magn. 36, 3238 (2000).

[26] M. Wun-Fogle, J. B. Restorff, and A. E. Clark, Magnetostriction of stress-annealed Fe-Ga and Fe-Ga-Al alloys under compressive and tensile stress, J. Intell. Mater. Syst. Struct. 17, 117 (2006).

[27] J.-H. Yoo, J. B. Restorff, M. Wun-Fogle, and A. B. Flatau, Induced magnetic anisotropy in stress-annealed Galfenol laminated rods, Smart Mater. Struct. 18, 104004 (2009).

[28] M. Wun-Fogle, J. B. Restorff, A. E. Clark, E. Dreyer, and E. Summers, Stress annealing of $\mathrm{Fe}-\mathrm{Ga}$ transduction alloys for operation under tension and compression, J. Appl. Phys. 97, 10M301 (2005).

[29] J. Atulasimha and A. B. Flatau, A review of magnetostrictive iron-gallium alloys, Smart Mater. Struct. 20, 043001 (2011).

[30] J.-H. Yoo, J. B. Restorff, M. Wun-Fogle, and A. B. Flatau, The effect of magnetic field annealing on single crystal iron gallium alloy, J. Appl. Phys. 103, 07B325 (2008).

[31] J. Steiner, A. Lisfi, T. Kakeshita, T. Fukuda, and M. Wuttig, Unique magnetostriction of $\mathrm{Fe}_{68.8} \mathrm{Pd}_{31.2}$ attributable to twinning, Sci. Rep. 6, 34259 (2016).

[32] Y. K. He, Y. J. Han, P. Stamenov, B. Kundys, J. M. D. Coey, C. B. Jiang, and H. B. Xu, Investigating non-Joulian magnetostriction, Nature (London) (to be published).

[33] P. Stamenov and J. M. D. Coey, Vector vibrating-sample magnetometer with permanent magnet flux source, J. Appl. Phys. 99, 08D912 (2006).

[34] J. B. Restorff, M. Wun-Fogle, K. B. Hathaway, A. E. Clark, T. A. Lograsso, and G. Petculescu, Tetragonal magnetostriction and magnetoelastic coupling in $\mathrm{Fe}-\mathrm{Al}, \mathrm{Fe}-\mathrm{Ga}, \mathrm{Fe}-\mathrm{Ge}, \mathrm{Fe}-\mathrm{Si}$, FeGa-Al, and Fe-Ga-Ge alloys, J. Appl. Phys. 111, 023905 (2012).

[35] R. H. Baughman, J. M. Shacklette, A. A. Zakhidov, and S. Stafström, Negative Poisson's ratios as a common feature of cubic metals, Nature (London) 392, 362 (1998).
[36] V. Z. C. Paes and D. H. Mosca, Magnetostrictive contribution to Poisson ratio of galfenol, J. Appl. Phys. 114, 123915 (2013).

[37] J.-H. Yoo and A. B. Flatau, Measurement of field-dependence elastic modulus of iron-gallium alloy using tensile test, J. Appl. Phys. 97, 10M318 (2005).

[38] A. E. Clark, K. B. Hathaway, M. Wun-Fogle, J. B. Restorff, T. A. Lograsso, V. M. Keppens, G. Petculescu, and R. A. Taylor, Extraordinary magnetoelasticity and lattice softening in bcc $\mathrm{Fe}$ Ga alloys, J. Appl. Phys. 93, A621 (2003).

[39] Q. Xing, Y. Du, R. J. McQueeney, and T. A. Lograsso, Structural investigations of $\mathrm{Fe}-\mathrm{Ga}$ alloys: Phase relations and magnetostrictive behavior, Acta Mater. 56, 4536 (2008).

[40] Y. Zhou, X. L. Wang, W. Wang, Y. Liu, Y. Du, Y. C. Niu, and L. Q. Liu, Magnetostrictive properties of directional solidification $\mathrm{Fe}_{82} \mathrm{Ga}_{9} \mathrm{Al}_{9}$ alloy, J. Appl. Phys. 111, 07A332 (2012).

[41] R. Skomski, A. Kashyap, J. Zhou, and D. J. Sellmyer, Anisotropic exchange, J. Appl. Phys. 97, $10 \mathrm{~B} 302$ (2005).

[42] R. P. Beardsley, D. E. Parkes et al., Effect of lithographicallyinduced strain relaxation on the magnetic domain configuration in microfabricated epitaxially grown $\mathrm{Fe}_{81} \mathrm{Ga}_{19}$, Sci. Rep. 7, 42107 (2017).

[43] S. Guruswamy, T. V. Jayaraman, R. P. Corson, G. Garside, and S. Thuanboon, Short range ordering and magnetostriction in $\mathrm{Fe}-\mathrm{Ga}$ and other Fe alloy single crystals, J. Appl. Phys. 104, 113919 (2008).

[44] S. F. Xu, H. P. Zhang, W. Q. Wang, S. H. Guo, W. Zhu, Y. H. Zhang, X. L. Wang, D. L. Zhao, J. L. Chen, and G. H. Wu, Improved magnetostriction in $\mathrm{Fe}_{83} \mathrm{Ga}_{17}$ alloy by tensile-stress annealing treatment, J. Alloys Compd. 469, 203 (2009).

[45] N. Srisukhumbowornchai and S. Guruswamy, Large magnetostriction in directionally solidified $\mathrm{FeGa}$ and $\mathrm{FeGaAl}$ alloys, J. Appl. Phys. 90, 5680 (2001). 